\title{
Development of a Community Based Web-Mobile Platform (CBWMP) for diabetes care in Sri Lanka
}

\author{
Dr. Nishan Siriwardena MD, MSc \\ Medical Officer (Health Informatics), Health Information and Research Unit, National Cancer Institute Mahargama, \\ Sri Lanka \\ E-Mail address: siriwardenanishan@gmail.com
}

Dr. Sudarshana Wickramasinghe MBBS, MSc

Medical Officer (Health Informatics), Teaching Hospital Peradeniya, Sri Lanka

E-Mail address: wickramasinghewas@gmail.com

Dr. Dussantha Perera BDS, MSc

Medical Officer (Health Informatics), Prison Hospital Welikada, Sri Lanka

E-Mail address:dussanthaperera@gmail.com

Dr. Rohana Basil Marasinghe MBBS, MPhil, $\mathrm{PhD}$

Senior Lecturer, Department of Medical Education and Health Sciences, Faculty of Medical Sciences, University of Sri Jayawardenepura, Sri Lanka

E-Mail address: rohanabm@yahoo.com

Dr. Lanka Prasad Katulanda MBBS, MD, DPhil

Senior Lecturer, Diabetes Research Unit, Department of Clinical Medicine, University of Colombo, Sri Lanka E-Mail address: pkatulanda@yahoo.com

Dr. Roshan Hewapathirana MBBS, MSc, MIEEE, SCMAD

Research Fellow, Department of Informatics, Faculty of Mathematics and Natural Sciences, University of Oslo, Norway

E-Mail address: roshanhewapathirana@gmail.com

Sri Lanka Journal of Bio-Medical Informatics 2012;3(4):115-122

doi: http://dx.doi.org/10.4038/sljbmi.v3i4.5504

\begin{abstract}
Diabetes is a chronic disease with no permanent cure. Sri Lanka is placed among the countries with the highest diabetes prevalence rates in the world (ie. 2.8 million Sri Lankans have diabetes or pre-diabetes, and most importantly, a significant proportions of the population is yet to be diagnosed). Patients with diabetes need lifelong care to prevent complications which further impose a significant burden on the country's expenditure on healthcare. Moreover, patients need to maintain constant contact with the healthcare provider for the optimal management of diabetes. However, such arrangement is often costly and time consuming and therefore it ultimately aggravates the burden to patients, the healthcare system and the economy.

With the development of telecommunication technologies, Telemedicine (i.e. the use of Information Communication Technology to provide healthcare at a distance) has gained attention. Telemedicine can enhance communication between patient and healthcare provider without needing physical presence in one place. Telemedicine can link healthcare professionals from different corners of the globe to share knowledge and expertise. Moreover, evidence is surfacing to suggest that the telemedicine would be a viable alternative to conventional care.

This article showcases a Sri Lankan study which describes the development of a Telemedicine system for Sri Lanka - Community Based Web-Mobile Platform (CBWMP). The concept of the platform is to maintain an electronic
\end{abstract}


Personal Health Record (e-PHR) in order to provide communication between different parties to optimise patient health information flow and also to coordinate the continuity of care at minimal cost. The CBWMP - integrated mobile phones and e-PHR - is capable of delivering diabetes education, co-ordinating effective management, and screening diabetes status. To avoid any cultural marginalisation, all the services can be accessed in the user's preferred native language in Sri Lanka viz. Sinhala, Tamil, and English.

Keywords - e-PHR; telemedicine; mobile health; diabetes

\section{Introduction}

Diabetes is not a new disease. It has affected people around the globe for centuries. However, recent studies have shown that, mainly due to socio economic changes, the disease has now reemerged with a vengeance ${ }^{(1,2)}$. The worldwide pandemic of diabetes accounts for 346 million cases and nearly $80 \%$ of those diagnosed live in developing countries ${ }^{(2,3)}$.

Sri Lanka is placed among the countries with the highest diabetes prevalence rates in the world. As rapid industrialisation and modernisation changes the lifestyles of its population, Sri Lanka faces an increased incidence of diabetes. This is a cause for concern in view of the fact that South Asians are more vulnerable to type 2 diabetes $^{(4)}$. According to the Sri Lanka Diabetes and Cardiovascular Study (SLDCS) conducted by the Diabetes Research Unit of the University of Colombo and the Oxford Centre for Diabetes Endocrinology and Metabolism UK, 20\% of Sri Lankan adults have either diabetes or pre-diabetes while $33 \%$ of patients with diabetes are undiagnosed $^{(2)}$. It further estimated that 2.8 million Sri Lankans have diabetes and a significant proportion of them are yet to be diagnosed ${ }^{(2)}$.

Chowdhury and colleagues predicted that the Sri Lankan population is aging rapidly, and in the future the rate of aging will surpass almost all other developing countries ${ }^{(3)}$. This report also postulated that the above 65 year old population is expected to double by $2035^{(3)}$. It is obvious that the impact of diabetes on this segment of the population would be a significant burden for Sri Lanka. Owing to the chronic nature of the disease and complications that are associated with diabetes, patients carry an increased risk of morbidity, mortality and a decreased productive existence. At the same time, increased health expenditure together with loss of productive population would certainly hamper the development of the country. This impending crisis calls for innovative strategies.

Looking beyond the conventional wisdom and re-application of age-old preventive tools, evidence suggests that Telemedicine is a viable alternative to conventional care ${ }^{(5,6)}$ which could guide Sri Lanka to meet this challenge. Telemedicine can be defined as the delivery of healthcare using ICT at a distance. Uses of telemedicine can vary; from a videoconference between a patient and a doctor, to a text massage between a junior doctor and a senior, electronic medication reminders, to storage and forwarding of digital photography for diagnosis, to telemonitoring and sending feedback to patients by the attending physician. Telephone and wireless technology can be used to effectively transfer data from medical devices to a healthcare provider (eg. sending blood glucose value to medical personnel for opinion or remote monitoring of surgically implanted devices such as pacemakers and cardiac defibrillators for clinical or device assessment). Tele-health can empower patients to manage their own health by providing customised information directly from their healthcare provider. 
Exploration of new avenues of healthcare delivery to the needy necessitates evidence based scientific evaluation of available alternatives. Widespread availability of mobile phones, Internet, and steady reduction in costs, related to communication through these media make it an attractive and a reliable tool. Therefore, Telemedicine can provide tools to caregivers and patients to improve communication.

In summary, appropriate use of Telemedicine can play a decisive role in efficient and effective healthcare delivery. The success or the failure of Telemedicine interventions is a balance between the usability and the ability of such applications to meet the disease specific requirements and cost effectiveness. The decision to initiate a telemedicine application should always accompany careful planning and proper evaluation of ground realities.

\section{Method}

The concept of a 'community base web mobile-ICT platform' developed for suicide prevention ${ }^{(6)}$ was used for the study whereby the Mobile-ICT platform for a 'diabetic clinic' was customised ${ }^{(7)}$ to expand and involve the state owned mobile telecommunication provider, Sri Lanka Telecom Mobitel to develop the current CBWMP. Moreover, the development of the CBWMP was carried out in collaboration with the Diabetes Research Unit, Faculty of Medicine University of Colombo.

Steps adopted to design and develop CBWMP were as follows:

1. Analysis of the current clinical management protocols.

2. Review of the scientific literature to analyse current screening methods and management of diabetes mellitus ${ }^{(8)}$.

3. A series of structured interviews were conducted with a consultant endocrinologist/diabetologist to understand the screening and management options suitable for the Sri Lankan population.

4. Analysis of the consensus algorithm for diabetes screening and care was analysed.

5. Identifying improvements to be made in developing the CBWMP.

\section{Results}

Results can be shown as two components namely web platform and mobile application. The Mobile phone component of the application can be accessed through the telephone number +9471691094. The home page of the web access is shown in the Figure 1. (URL: www.mhealth.mobitel.1k.). The mobile application can be accessed by calling 071-691-0941.

The telemedicine application was designed to operate on both the mobile phone and the web platform. This application has the following components.

(a) Type 2 diabetes screening module. 
(b) Automated response system.

(c) Diabetes information component.

(d) Life style advice sub component.

(e) Self monitored blood glucose component.

(f) Electronic personal health record.

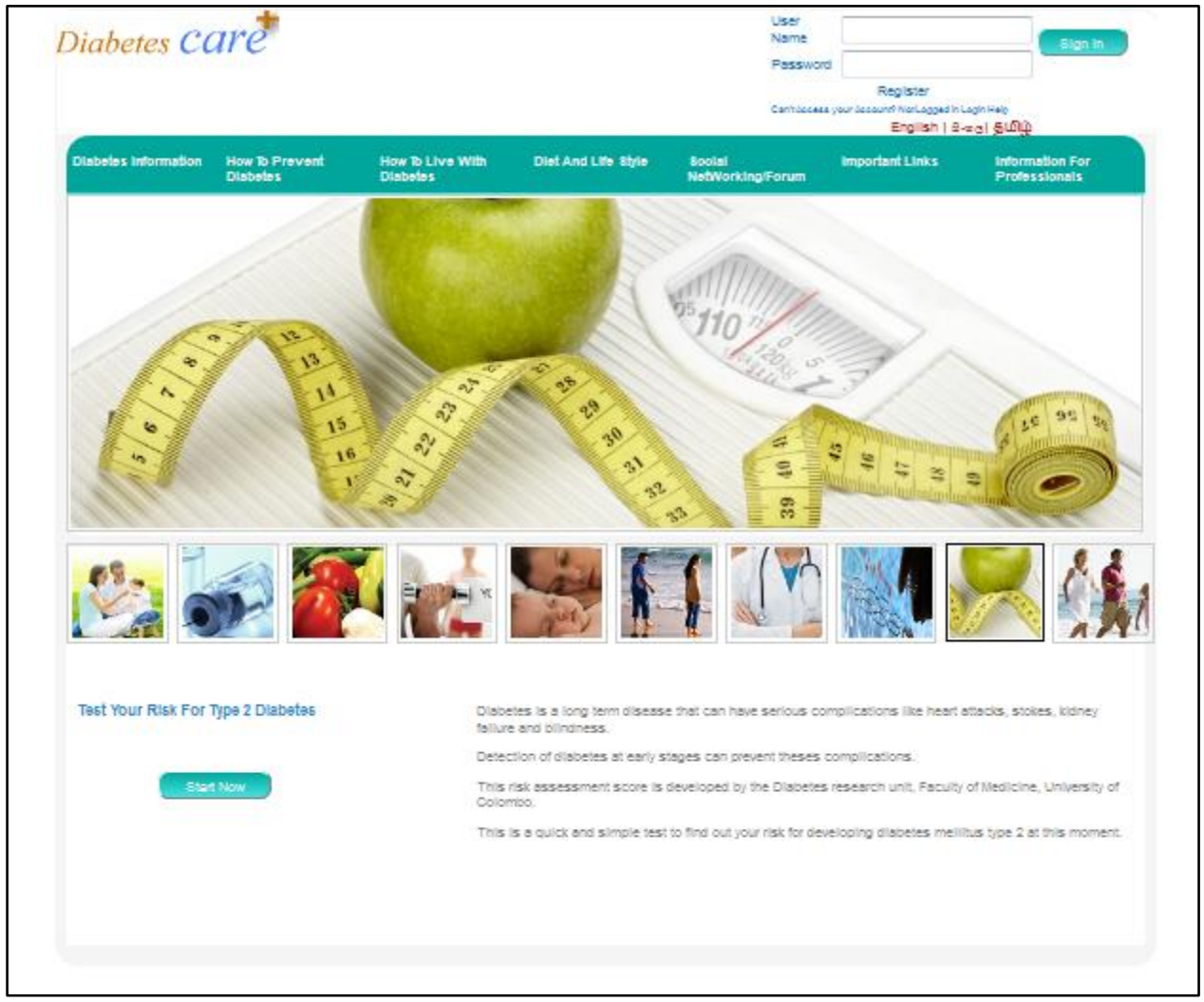

Figure 1. Home page

\section{(a) Type 2 diabetes screening module}

In the mobile platform it utilises interactive voice response (IVR) technology where the user interacts with the database using keypad of the mobile phone in response to voice commands. At the end point, after a series of interactions, the user will receive a diabetes risk report, list of modifiable risk factors and measures to manage those risk factors via voice call and SMSs. In the web platform it displays these massages to the user (See Figure 2).

\section{(b) Automated response system}

This module employs an electronic library of massages and a decision support system to facilitate communication. Massaging system is designed to operate at pre decided intervals (daily, monthly and once in three months) and it is capable of sending real time massages as well. 


\section{(c) Diabetes information component}

This module provides users with information on diabetes namely what is glucose, what is diabetes, what is insulin, the relationship of blood glucose and diabetes etc. Recommendations to prevent diabetes, living with diabetes, food and life style, diabetes communities as well as important contacts are also provided in this module. Figure 3 is a screenshot of such advices.

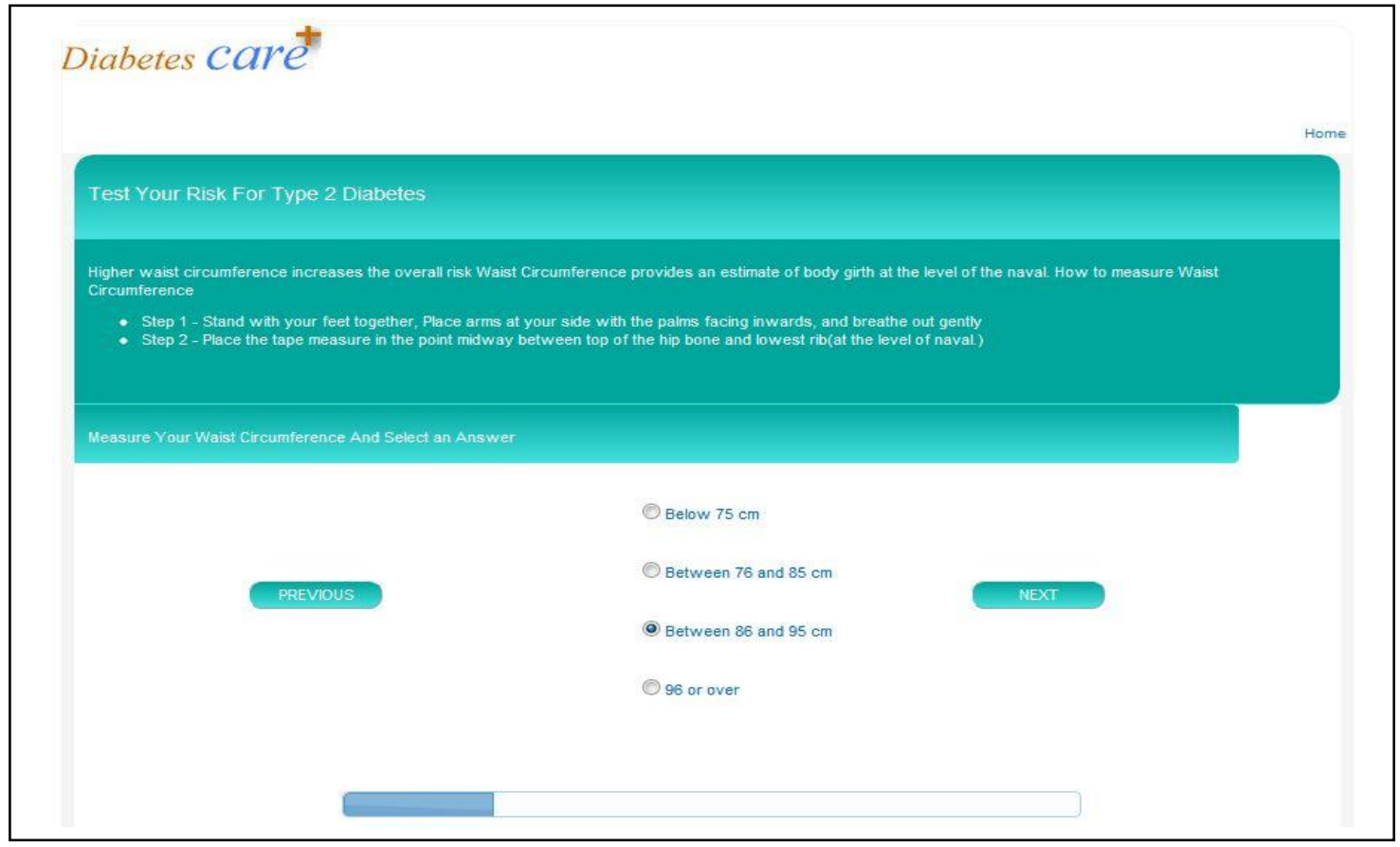

Figure 2. Diabetes screening module

\section{(d) Life style advice sub component}

This module is also similar to the information module but specifically focuses on life style modifications.

\section{(e) Self monitored blood glucose module}

This module is capable of storing and retrieval of blood glucose values together with their date and time taken. Moreover, this module provides a forum for different health care workers to interact with patient improving communication with each other (Figure 4).

\section{(f) Electronic personal health record (e-PHR)}

e-PHR is the backbone of the module which provide the platform to store and retrieval of patents personal health record.

This application is capable of delivering diabetes education, coordinating effective management and screening diabetic status. People could access services in their native language. Spiral 
system development methodology (where there is series of repetition of activities taking place) was adopted in Designing and development of the application.

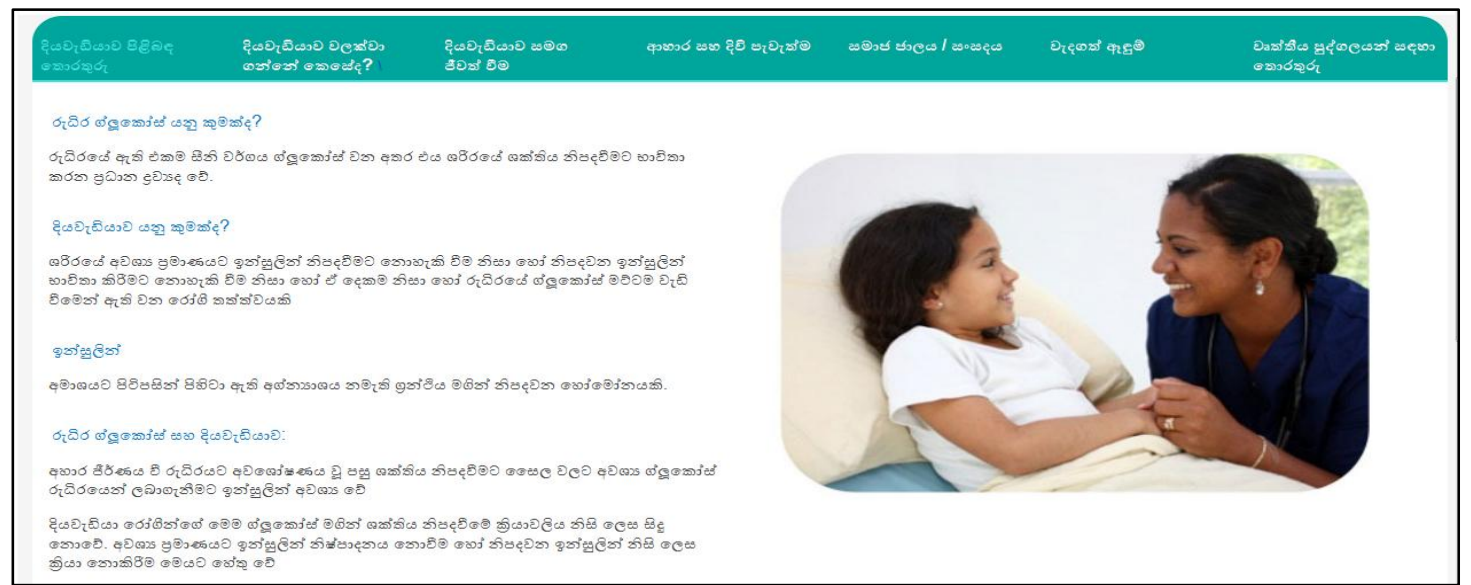

Figure 3. Life style advice sub component

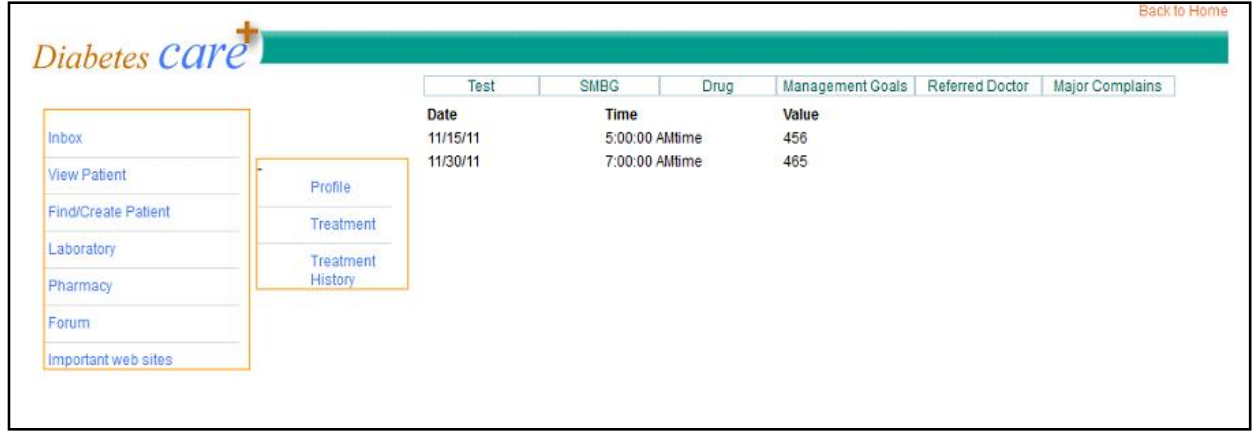

Figure 4. Self monitored blood glucose module

Users (patients) can utilise the diabetes education component by using mobile phone and Internet. Users from any part of the country can retrieve information in their preferred language at any time. They have the opportunity to assess the risk of diabetes and to obtain investigation suggestions to verify the assessment. A web application user can access selected reliable information content through this application.

Patients can maintain their e-PHR with updated disease management information and investigation reports and could authorise access to his care history when seeking care from anywhere in the country. They can transfer self-monitored blood glucose values to the attending physician through the telemedicine application and receive feedback. Patients can receive alerts and reminders to facilitate good compliance and desirable control of the disease. They can participate in individual treatment setting processes with attending physicians to achieve optimal management. Emergencies could be prevented by facilitating instant access to doctors through a mobile phones and Internet. Lifesaving health tips can be delivered to patients based on outputs from the diabetes decision support system. 


\section{Discussion and conclusion}

When considering deployment of telemedicine applications to a wider section of Sri Lankan society, one has to overcome the major obstacles of low ICT literacy and Internet penetration. A major portion of ICT literate segment constitute the educated youth, while diabetes prevalence is high among the elderly. While Internet penetration is still at a lower level in rural areas, undiagnosed diabetes reigns high among the rural population. It draws a bleak picture of services not reading the neediest segment of the population. Even though a higher mobile phone penetration could address this inequality to some extent, technical limitations of mobile devices prevents the user from reaping the full benefits of telemedicine applications. Indefinite legal status is the major limiting factor affecting wider deployment of technology of this nature. The authors expect that with the implementation of the proposed e-Government policy, necessary amendments to the existing legal structure would create an environment conducive for the development of mHealth. Although, pilot level evaluation of CBWMP gives promising results, the community level evaluation of the system is still needed.

\section{Acknowledgements}

We acknowledge funding from the Postgraduate Institute of Medicine, University of Colombo and technological support from SLT Mobitel Sri Lanka (Pvt) Ltd.

\section{Reference}

1. Wild S, Roglic G, Green A, Sicree R, King H. Global prevalence of diabetes: estimates for the year 2000 and projections for 2030. Diabetes Care. 2004; 1047-53.

doi: http://dx.doi.org/10.2337/diacare.27.5.1047

2. Katulanda P, Constantine G, Mahesh J, Sheriff R, Seneviratne R. Prevalence and projections of diabetes and pre-diabetes in adults in Sri Lanka-Sri Lanka Diabetes, Cardiovascular Study (SLDCS). Diabetic Medicine. 2008; 25: 1062-69. Accessed in November 2011. Available from: http://www.who.int/mediacentre/factsheets/fs312/en/index.html doi: http://dx.doi.org/10.1111/j.1464-5491.2008.02523.x

3. Chowdhury T, Grace C, Kopelman P. Preventing diabetes in south Asians, Too little action and too late. BMJ. 2003; 8(327):1059-60.

doi: http://dx.doi.org/10.1136/bmj.327.7423.1059

4. West SP, Lagua C, Trief PM, Izquierdo R, Weinstock RS. Goal setting using telemedicine in rural underserved older adults with Diabetes: Experiences from the informatics for diabetes education and telemedicine project. Telemedicine Journal and e-Health. 2010, 16(4):405-16. doi: http://dx.doi.org/10.1089/tmj.2009.0136

5. Weinstock RS, Teresi JS, Goland R. Glycemic control and health disparities in older ethnically diverse underserved adults with diabetes. Diabetes Care. 2011; 34: 274-9. doi: http://dx.doi.org/10.2337/dc10-1346 
6. Marasinghe RB, Edirippulige S, Kavanagh D, Smith A, Jiffry MTM. Effect of mobile phonebased psychotherapy in suicide prevention: a randomized controlled trial in Sri Lanka.

Journal of Telemedicine and Telecare. 2012.

doi: http://dx.doi.org/10.1258/jtt.2012.SFT107

7. Kumara MHSP, Weerasingha DDPM, Siriwardene TMDASK, Perera MDR, Marasinghe RB. Development of "Mobile Clinic", a mobile phone based software to improve compliance in managing diabetes. Proceedings of the 30th Anniversary Academic Sessions of the Post Graduate Institute of Medicine. Colombo, Sri Lanka. 2011; 37

8. Siriwardena NLSA, Wickramasinghe SWA, Perera DKL, Marasinghe RB, Katulanda P, Hewapathirana R. A review of telemedicine interventions in diabetes care. Journal of Telemedicine and Telecare 2012;18(3):164-8.

doi: http://dx.doi.org/10.1258/jtt.2012.SFT110 